\title{
de Rham and Dolbeault cohomology of solvmanifolds with local systems
}

\author{
HISASHI KASUYA
}

Let $G$ be a simply connected solvable Lie group with a lattice $\Gamma$ and the Lie algebra $\mathfrak{g}$ and a representation $\rho: G \rightarrow G L\left(V_{\rho}\right)$ whose restriction on the nilradical is unipotent. Consider the flat bundle $E_{\rho}$ given by $\rho$. By using "many" characters $\{\alpha\}$ of $G$ and "many" flat line bundles $\left\{E_{\alpha}\right\}$ over $G / \Gamma$, we show that an isomorphism

$$
\bigoplus_{\{\alpha\}} H^{*}\left(\mathfrak{g}, V_{\alpha} \otimes V_{\rho}\right) \cong \bigoplus_{\left\{E_{\alpha}\right\}} H^{*}\left(G / \Gamma, E_{\alpha} \otimes E_{\rho}\right)
$$

holds. This isomorphism is a generalization of the well-known fact: "If $G$ is nilpotent and $\rho$ is unipotent then, the isomorphism $H^{*}\left(\mathfrak{g}, V_{\rho}\right) \cong H^{*}\left(G / \Gamma, E_{\rho}\right)$ holds". By this result, we construct an explicit finite-dimensional cochain complex which compute the cohomology $H^{*}\left(G / \Gamma, E_{\rho}\right)$ of solvmanifolds even if the isomorphism $H^{*}\left(\mathfrak{g}, V_{\rho}\right) \cong H^{*}\left(G / \Gamma, E_{\rho}\right)$ does not hold. For Dolbeault cohomology of complex parallelizable solvmanifolds, we also prove an analogue of the above isomorphism result which is a generalization of computations of Dolbeault cohomology of complex parallelizable nilmanifolds. By this isomorphism, we construct an explicit finite-dimensional cochain complex which compute the Dolbeault cohomology of complex parallelizable solvmanifolds.

\section{Background and main results}

\subsection{Background}

We have nice theorem for de Rham cohomology of nilmanifolds with local systems.

1991 Mathematics Subject Classification. Primary: 17B30, 17B56, 22E25, 53C30, Secondary: 32M10, 55N25, 58A12

Key words and phrases. de Rham cohomology, local system, Lie algebra cohomology, Dolbeault cohomology, solvmanifold. 
Theorem 1.1 (due to [12] or [15]). Let $N$ be a simply connected real nilpotent Lie group and $\mathfrak{n}$ the Lie algebra of $N$. Suppose $N$ has a lattice $\Gamma$. Let $\rho: N \rightarrow G L\left(V_{\rho}\right)$ be a finite-dimensional unipotent representation. We define the flat bundle $E_{\rho}=\left(N \times V_{\rho}\right) / \Gamma$ given by the equivalent relation $(\gamma g, \rho(\gamma) v) \cong(g, v)$ for $g \in N, v \in V_{\rho}, \gamma \in \Gamma$. Consider the cochain complex $\bigwedge \mathfrak{n}_{\mathbb{C}}^{*} \otimes V_{\rho}$ of Lie algebra (see [13]) and the canonical inclusion

$$
\bigwedge \mathfrak{n}_{\mathbb{C}}^{*} \otimes V_{\rho} \rightarrow A^{*}\left(N / \Gamma, E_{\rho}\right) .
$$

Then this inclusion induces a cohomology isomorphism

$$
H^{*}\left(\mathfrak{n}, V_{\rho}\right) \cong H^{*}\left(N / \Gamma, E_{\rho}\right)
$$

Some researchers tried to extend Theorem 1.1 for solvmanifolds. In fact, it is proved that for a simply connected solvable Lie group $G$ with the Lie algebra $\mathfrak{g}$ admitting a lattice $\Gamma$ and a representation $\rho: G \rightarrow G L\left(V_{\rho}\right)$, if:

(H) ([6]) The representation $\rho \oplus \mathrm{Ad}$ is triangular or,

(M) ([11]) The two images $(\rho \oplus \operatorname{Ad})(G)$ and $(\rho \oplus \operatorname{Ad})(\Gamma)$ have same Zariski-closure in $G L\left(V_{\rho}\right) \times \operatorname{Aut}\left(\mathfrak{g}_{\mathbb{C}}\right)$,

then the isomorphism $H^{*}\left(\mathfrak{g}, V_{\rho}\right) \cong H^{*}\left(G / \Gamma, E_{\rho}\right)$ holds. However, in general the isomorphism $H^{*}\left(\mathfrak{g}, V_{\rho}\right) \cong H^{*}\left(N / \Gamma, E_{\rho}\right)$ does not hold.

As an Analogue of Theorem 1.1 we have the following theorem for Dolbeault cohomology of complex parallelizable nilmanifolds.

Theorem 1.2 (due to [16]). Let $N$ be a simply connected complex nilpotent Lie group and $\mathfrak{n}$ the Lie algebra (as a complex Lie algebra) of N. Suppose $N$ has a lattice $\Gamma$. Let $\sigma: N \rightarrow G L\left(V_{\sigma}\right)$ be a finite-dimensional holomorphic unipotent representation. We also consider the anti-holomorphic representation $\bar{\sigma}: N \rightarrow G L\left(V_{\bar{\sigma}}\right)$. Define the flat holomorphic vector bundle $L_{\bar{\sigma}}=(N \times$ $\left.V_{\bar{\sigma}}\right) / \Gamma$ over $G / \Gamma$ given by the equivalent relation $(\gamma g, \bar{\sigma}(\gamma) v) \cong(g, v)$ for $g \in N, v \in V_{\sigma}, \gamma \in \Gamma$. We consider the Dobeault complex $\left(A^{*, *}\left(N / \Gamma, L_{\bar{\sigma}}\right), \bar{\partial}\right)$. We regard $\bigwedge \mathfrak{n}^{*} \otimes V_{\sigma}$ as the subcomplex of $\left(A^{0, *}\left(N / \Gamma, L_{\bar{\sigma}}\right), \bar{\partial}\right)$ which consists of the left-invariant "anti"-holomorphic forms with values in $L_{\bar{\sigma}}$. Then the inclusion

$$
\bigwedge \mathfrak{n}^{*} \otimes V_{\sigma} \rightarrow A^{0, *}\left(N / \Gamma, L_{\bar{\sigma}}\right)
$$

induces a cohomology isomorphism

$$
H^{*}\left(\mathfrak{n}, V_{\sigma}\right) \cong H_{\bar{\partial}}^{0, *}\left(N, L_{\bar{\sigma}}\right)
$$


Hence since $N / \Gamma$ is complex parallelizable, we have an isomorphism

$$
\bigwedge \mathbb{C}^{\operatorname{dim} N} \otimes H^{*}\left(\mathfrak{n}, V_{\sigma}\right) \cong H_{\bar{\partial}}^{*, *}\left(N, L_{\bar{\sigma}}\right)
$$

It is desired that Theorems 1.1 and 1.2 are generalized for solvmanifolds and we can compute the de Rham and Dolbeault cohomology of solvmanifolds even if the isomorphism $H^{*}\left(\mathfrak{g}, V_{\rho}\right) \cong H^{*}\left(G / \Gamma, E_{\rho}\right)\left(\operatorname{resp} . \wedge \mathbb{C}^{\operatorname{dim} N} \otimes\right.$ $\left.H^{*}\left(\mathfrak{n}, V_{\sigma}\right) \cong H_{\bar{\partial}}^{*, *}\left(N, L_{\bar{\sigma}}\right)\right)$ does not holds.

\subsection{Main results}

The first purpose of this paper is to show new-type cohomology isomorphism theorems for solvmanifolds which are generalizations of Theorems 1.1 and 1.2. These analogous each other. We consider the "many" characters of $G$ and "many" line bundles over $G / \Gamma$. In this paper, we prove:

Theorem 1.3. Let $G$ be a simply connected real solvable Lie group with a lattice $\Gamma$ and $\mathfrak{g}$ the Lie algebra of $G$. Let $N$ be the nilradical (i.e., maximal connected nilpotent normal subgroup) of $G$. Let $\mathcal{A}_{(G, N)}=\left\{\alpha \in \operatorname{Hom}\left(G, \mathbb{C}^{*}\right)\right.$ $\left.\mid \alpha_{\left.\right|_{N}}=1\right\}$ and $\mathcal{A}_{(G, N)}(\Gamma)$ the set $\left\{E_{\alpha}\right\}$ of all the isomorphism classes of flat line bundles given by $\left\{V_{\alpha}\right\}_{\alpha \in \mathcal{A}_{(G, N)}}$. Let $\rho: G \rightarrow G L\left(V_{\rho}\right)$ be a representation. For the nilradical $N$ of $G$, we assume that the restriction $\rho_{\left.\right|_{N}}$ is a unipotent representation. We consider the direct sum

$$
\bigoplus_{\alpha \in \mathcal{A}_{(G, N)}} \bigwedge \mathfrak{g}_{\mathbb{C}}^{*} \otimes V_{\alpha} \otimes V_{\rho}
$$

of the Lie algebra cochain complexes. We also consider the direct sum

$$
\bigoplus_{E_{\alpha} \in \mathcal{A}_{(G, N)}(\Gamma)} A^{*}\left(G / \Gamma, E_{\alpha} \otimes E_{\rho}\right)
$$

Then the inclusion

$$
\bigoplus_{\alpha \in \mathcal{A}_{(G, N)}} \bigwedge \mathfrak{g}_{\mathbb{C}}^{*} \otimes V_{\alpha} \otimes V_{\rho} \rightarrow \bigoplus_{E_{\alpha} \in \mathcal{A}_{(G, N)}(\Gamma)} A^{*}\left(G / \Gamma, E_{\alpha} \otimes E_{\rho}\right)
$$

induces a cohomology isomorphism

$$
\bigoplus_{\alpha \in \mathcal{A}_{(G, N)}} H^{*}\left(\mathfrak{g}, V_{\alpha} \otimes V_{\rho}\right) \cong \bigoplus_{E_{\alpha} \in \mathcal{A}_{(G, N)}(\Gamma)} H^{*}\left(G / \Gamma, E_{\alpha} \otimes E_{\rho}\right)
$$


We also prove:

Theorem 1.4. Let $G$ be a simply connected complex solvable Lie group with a lattice $\Gamma$ and $\mathfrak{g}$ the Lie algebra (as a complex Lie algebra) of $G$. Let $N$ be the nilradical of $G$. Let $\mathcal{B}_{(G, N)}=\left\{\alpha \in \operatorname{Hom}_{\text {hol }}\left(G, \mathbb{C}^{*}\right) \mid \alpha_{\left.\right|_{N}}=1\right\}$ and $\mathcal{B}_{(G, N)}(\Gamma)$ the set $\left\{L_{\bar{\alpha}}\right\}$ of all the isomorphism classes of holomorphic line bundles given by $\left\{V_{\bar{\alpha}}\right\}_{\alpha \in \mathcal{B}_{(G, N)}}$. Let $\sigma: G \rightarrow G L\left(V_{\sigma}\right)$ be a holomorphic representation. For the nilradical $N$ of $G$, we assume that the restriction $\sigma_{\left.\right|_{N}}$ is a unipotent representation. We consider the direct sum

$$
\bigoplus_{\alpha \in \mathcal{B}_{(G, N)}} \bigwedge \mathfrak{g}^{*} \otimes V_{\alpha} \otimes V_{\sigma}
$$

of the Lie algebra cochain complexes. We also consider the direct sum

$$
\bigoplus_{L_{\bar{\alpha}} \in \mathcal{B}_{(G, N)}(\Gamma)} A^{0, *}\left(G / \Gamma, L_{\bar{\alpha}} \otimes L_{\bar{\sigma}}\right)
$$

of Dolbeault complexes.

Then the inclusion

$$
\bigoplus_{\alpha \in \mathcal{B}_{(G, N)}} \bigwedge \mathfrak{g}^{*} \otimes V_{\alpha} \otimes V_{\sigma} \rightarrow \bigoplus_{L_{\bar{\alpha}} \in \mathcal{B}_{(G, N)}(\Gamma)} A^{0, *}\left(G / \Gamma, L_{\bar{\alpha}} \otimes L_{\bar{\sigma}}\right)
$$

induces a cohomology isomorphism

$$
\bigoplus_{\alpha \in \mathcal{B}_{(G, N)}} H^{*}\left(\mathfrak{g}, V_{\alpha} \otimes V_{\sigma}\right) \cong \bigoplus_{L_{\bar{\alpha}} \in \mathcal{B}_{(G, N)}(\Gamma)} H^{0, *}\left(G / \Gamma, L_{\bar{\alpha}} \otimes L_{\bar{\sigma}}\right)
$$

Remark 1. The correspondence $\mathcal{A}_{(G, N)} \rightarrow \mathcal{A}_{(G, N)}(\Gamma) \quad\left(\operatorname{resp} . \quad \mathcal{B}_{(G, N)} \rightarrow\right.$ $\left.\mathcal{B}_{(G, N)}(\Gamma)\right)$ is not 1 to 1 . This remark is very important for the case the isomorphism $H^{*}\left(\mathfrak{g}, V_{\rho}\right) \cong H^{*}\left(G / \Gamma, E_{\rho}\right)\left(\operatorname{resp} . H^{*}\left(\mathfrak{g}, V_{\sigma}\right) \cong H^{0, *}\left(G / \Gamma, L_{\bar{\sigma}}\right)\right)$ does not hold.

The second purpose of this paper is to construct a explicit finite- dimensional cochain complex which compute the de Rham cohomology $H^{*}(G / \Gamma$, $\left.E_{\rho}\right)$ and the Dolbeault cohomology $H^{0, *}\left(G / \Gamma, L_{\bar{\sigma}}\right)$ using Theorems 1.3 and 1.4. We prove:

Theorem 1.5. Let $G$ be a simply connected real (resp complex) solvable Lie group and $\mathfrak{g}$ the Lie algebra of $G$. Define $\mathcal{A}_{(G, N)}\left(\right.$ resp. $\left.\mathcal{B}_{(G, N)}\right)$ as in Theorem 1.3 (resp Theorem 1.4). Let $\rho: G \rightarrow G L\left(V_{\rho}\right)$ (resp. $\sigma: G \rightarrow G L\left(V_{\sigma}\right)$ ) be a 
representation with the assumption of Theorem 1.3 (resp. Theorem 1.4). We consider the direct sum

$$
\bigoplus_{\alpha \in \mathcal{A}_{(G, N)}} \bigwedge \mathfrak{g}_{\mathbb{C}}^{*} \otimes V_{\alpha} \otimes V_{\rho}
$$

(resp.

$$
\bigoplus_{\alpha \in \mathcal{B}_{(G, N)}} \bigwedge \mathfrak{g}^{*} \otimes V_{\alpha} \otimes V_{\sigma}
$$

) of the Lie algebra cochain complexes.

Then there exists a finite-dimensional subcomplex

$$
A^{*} \subset \bigoplus_{\alpha \in \mathcal{A}_{(G, N)}} \bigwedge \mathfrak{g}_{\mathbb{C}}^{*} \otimes V_{\alpha} \otimes V_{\rho}
$$

(resp.

$$
B^{*} \subset \bigoplus_{\alpha \in \mathcal{B}_{(G, N)}} \bigwedge \mathfrak{g}^{*} \otimes V_{\alpha} \otimes V_{\sigma}
$$

) such that the inclusion induces a cohomology isomorphism.

By Theorem 1.3 (resp. Theorem 1.4), we have the inclusion

$$
\iota: A^{*} \rightarrow \bigoplus_{E_{\alpha} \in \mathcal{A}_{(G, N)}(\Gamma)} A^{*}\left(G / \Gamma, E_{\alpha} \otimes E_{\rho}\right)
$$

(resp.

$$
\iota: B^{*} \rightarrow \bigoplus_{L_{\bar{\alpha}} \in \mathcal{A}_{(G, N)}(\Gamma)} A^{0, *}\left(G / \Gamma, L_{\bar{\alpha}} \otimes L_{\bar{\sigma}}\right)
$$

) inducing a cohomology isomorphism. Hence we have:

Corollary 1.6. Let $A_{\Gamma}^{*}=\iota^{-1}\left(A^{*}\left(G / \Gamma, E_{\rho}\right)\right) \quad\left(\right.$ resp. $B_{\Gamma}^{*}=\iota^{-1}\left(A^{0, *}(G / \Gamma\right.$, $\left.\left.L_{\bar{\sigma}}\right)\right)$. Then we have an isomorphism

$$
H^{*}\left(A_{\Gamma}^{*}\right) \cong H^{*}\left(G / \Gamma, E_{\rho}\right)
$$

(resp.

$$
H^{*}\left(B_{\Gamma}^{*}\right) \cong H^{0, *}\left(G / \Gamma, L_{\bar{\sigma}}\right)
$$

and hence $\left.\bigwedge \mathbb{C}^{\operatorname{dim} G} \otimes H^{*}\left(B_{\Gamma}^{*}\right) \cong H^{*, *}\left(G / \Gamma, L_{\bar{\sigma}}\right)\right)$. 
Consider the adjoint representation Ad. Then the restriction $\operatorname{Ad}_{\left.\right|_{N}}$ is unipotent. Hence by the above cochain complex $A_{\Gamma}^{*}$, we can compute the cohomology $H^{*}\left(G / \Gamma, E_{\mathrm{Ad}}\right)$ on general solvmanifolds. The cohomology

$$
H^{*}\left(G / \Gamma, E_{\mathrm{Ad}}\right) \cong H^{*}(\Gamma, \mathrm{Ad})
$$

is important for studying the deformation of lattice $\Gamma$ in $G$.

\section{Preliminary: Jordan decompositions of representations}

Let $A \in G L_{n}(\mathbb{C})$. We denote by $A_{s}$ (resp. $A_{u}$ ) the semi-simple (resp. unipotent) part of $A$ for the Jordan decomposition (see [8] for the definition). We will use the following facts.

Lemma 2.1. Let $N$ be a simply connected nilpotent Lie group and $\varphi: N \rightarrow$ $G L\left(V_{\varphi}\right)$ a representation. Then the map $\varphi^{\prime}: N \ni g \rightarrow(\varphi(g))_{s}$ is also a representation (see [2]). Since $\varphi^{\prime}(N)$ is connected nilpotent group and consists of semi-simple elements, the Zariski-closure of $\varphi^{\prime}(N)$ is an algebraic torus (see $\left[8\right.$, Section 19]) and hence $\varphi^{\prime}$ is diagonalizable.

\section{Proof of Theorem 1.3}

\subsection{Cohomology of tori}

Let $A$ be a simply connected real abelian Lie group with a lattice $\Gamma$ and $\mathfrak{a}$ the Lie algebra of $A$.

Lemma 3.1. Let $\rho: A \rightarrow G L\left(V_{\rho}\right)$ be a representation. Suppose $\rho=\beta \otimes \phi$ such that $\beta$ is a character of $A$ and $\phi$ is a unipotent representation. Then we have:

If $\beta$ is non-trivial, then we have

$$
H^{*}\left(\mathfrak{a}, V_{\rho}\right)=0 .
$$

If the flat line bundle $E_{\beta}$ is non-trivial, then we have

$$
H^{*}\left(A / \Gamma, E_{\rho}\right)=0 .
$$


Proof. Suppose $\operatorname{dim} V_{\rho}=1$. Then if $\beta$ is non-trivial, we can show $H^{*}\left(\mathfrak{a}, V_{\rho}\right)=$ $H^{*}\left(\mathfrak{a}, V_{\beta}\right)=0$ by simple computation and if $E_{\beta}$ is non-trivial, then we have

$$
H^{*}\left(A / \Gamma, E_{\rho}\right)=H^{*}(\Gamma, \beta)=0
$$

by [10, Lemma 2.1].

In case $\operatorname{dim} V_{\sigma}=n>1$, by the triangulation of $\rho$, we have a $(n-1)$ dimensional $A$-submodule $V_{\rho^{\prime}}$ such that $V_{\rho} / V_{\rho^{\prime}}=V_{\beta}$. Then by the long exact sequence of cohomology of Lie algebra or group (see [13]), the lemma follows inductively.

Lemma 3.2. Let $\rho: A \rightarrow G L\left(V_{\rho}\right)$ be a representation. Then we have a basis of $V_{\rho}$ such that $\rho$ is represented by

$$
\rho=\bigoplus_{i=1}^{k} \alpha_{i} \otimes \phi_{i}
$$

for characters $\alpha_{i}$ of $G$ and unipotent representations $\phi_{i}$ of $G$.

Proof. For a character $\alpha$, we denote by $W_{\alpha}$ the subspace of $V_{\rho}$ consisting of the elements $w \in V_{\rho}$ such that for some positive integer $n$ we have $(\rho(a)-$ $\alpha(a) I)^{n} w=0$ for any $a \in A$. Since $A$ is abelian, we have a decomposition

$$
V_{\rho}=W_{\alpha_{1}} \oplus \cdots \oplus W_{\alpha_{k}}
$$

by generalized eigenspace decomposition of $\rho(a)$ for all $a \in A$. Let $\rho_{i}(a)=$ $(\rho(a))_{W_{\alpha_{i}}}$. Then we have $\rho=\rho_{1} \oplus \cdots \oplus \rho_{k}$. We have $\left(\rho_{i}(a)\right)_{s}=\alpha_{i} I$. Let $\phi_{i}(a)=\left(\rho_{i}(a)\right)_{u}$. By Lemma 2.1, $\phi_{i}$ is a unipotent representation and we have $\rho_{i}(a)=\left(\rho_{i}(a)\right)_{s}\left(\rho_{i}(a)\right)_{u}=\left(\alpha_{i} \otimes \phi_{i}\right)(a)$. Hence the Lemma follows.

Let $\left\{V_{\alpha}\right\}_{\alpha \in \operatorname{Hom}\left(A, \mathbb{C}^{*}\right)}$ be the set of all one-dimensional representations of $A$ and $\mathcal{H}(A / \Gamma)=\left\{E_{\beta}\right\}$ the set of all the isomorphism classes of flat line bundles given by $\left\{V_{\alpha}\right\}_{\alpha \in \operatorname{Hom}\left(A, \mathbb{C}^{*}\right)}$. We notice that the correspondence $\left\{V_{\alpha}\right\}_{\alpha \in \operatorname{Hom}\left(A, \mathbb{C}^{*}\right)} \rightarrow \mathcal{H}(A / \Gamma)$ is not injective. We consider the direct sums

$$
\bigoplus_{\alpha \in \operatorname{Hom}\left(A, \mathbb{C}^{*}\right)} \bigwedge \mathfrak{a}_{\mathbb{C}}^{*} \otimes V_{\alpha} \otimes V_{\rho}
$$

and

$$
\bigoplus_{E_{\alpha} \in \mathcal{H}(A / \Gamma)} A^{*}\left(A / \Gamma, E_{\alpha} \otimes E_{\rho}\right)
$$


Proposition 3.3. The inclusion

$$
\bigoplus_{\alpha \in \operatorname{Hom}\left(A, \mathbb{C}^{*}\right)} \bigwedge \mathfrak{a}_{\mathbb{C}}^{*} \otimes V_{\alpha} \otimes V_{\rho} \rightarrow \bigoplus_{E_{\alpha} \in \mathcal{H}(A / \Gamma)} A^{*}\left(A / \Gamma, E_{\alpha} \otimes E_{\rho}\right)
$$

induces a cohomology isomorphism.

Proof. Consider the decomposition

$$
\sigma=\bigoplus_{i=1}^{k} \alpha_{i} \otimes \phi_{i}
$$

as the above lemma. Then we have

$$
\bigoplus_{\alpha \in \operatorname{Hom}\left(A, \mathbb{C}^{*}\right)} \bigwedge \mathfrak{a}_{\mathbb{C}}^{*} \otimes V_{\alpha} \otimes V_{\rho}=\bigoplus_{\alpha \in \operatorname{Hom}\left(A, \mathbb{C}^{*}\right)} \bigwedge \mathfrak{a}^{*} \otimes \bigoplus_{i=1}^{k} V_{\alpha \alpha_{i}} \otimes V_{\phi_{i}}
$$

and

$$
\bigoplus_{E_{\alpha} \in \mathcal{H}(A / \Gamma)} A^{*}\left(A / \Gamma, E_{\alpha} \otimes E_{\rho}\right)=\bigoplus_{E_{\alpha} \in \mathcal{H}(A / \Gamma)} A^{*}\left(A / \Gamma, \bigoplus_{i=1}^{k} E_{\alpha} \otimes E_{\alpha_{i}} \otimes E_{\phi_{i}}\right) .
$$

By Theorem 1.1 and Lemma 3.1, we have

$$
\begin{aligned}
H^{*}\left(\bigoplus_{E_{\alpha} \in \mathcal{H}(A / \Gamma)} A^{*}\left(A / \Gamma, E_{\alpha} \otimes E_{\rho}\right)\right) & \cong H^{*}\left(A / \Gamma, \bigoplus_{i=1}^{k} E_{\phi_{i}}\right) \\
& \cong H^{*}\left(\mathfrak{a}, \bigoplus_{i=1}^{k} V_{\phi_{i}}\right) .
\end{aligned}
$$

By Lemma 3.1 we have

$$
H^{*}\left(\bigoplus_{\alpha \in \operatorname{Hom}\left(A, \mathbb{C}^{*}\right)} \bigwedge \mathfrak{a}_{\mathbb{C}}^{*} \otimes V_{\alpha} \otimes V_{\rho}\right) \cong H^{*}\left(\mathfrak{a}, \bigoplus_{i=1}^{k} V_{\phi_{i}}\right)
$$

Hence the proposition follows.

\subsection{Mostow bundle and spectral sequence}

Let $G$ be a simply connected solvable Lie group with a lattice $\Gamma$ and $\mathfrak{g}$ be the Lie algebra of $G$. Let $N$ be the nilradical of $G$. It is known that $\Gamma \cap N$ 
is a lattice of $N$ and $\Gamma / \Gamma \cap N$ is a lattice of the abelian Lie group $G / N$ (see $[15])$. The solvmanifold $G / \Gamma$ is a fibre bundle

$$
N / \Gamma \cap N=N \Gamma / \Gamma \longrightarrow G / \Gamma \longrightarrow G / N \Gamma=(G / N) /(\Gamma / \Gamma \cap N)
$$

over a torus with a nilmanifold $N / \Gamma \cap N$ as fibre. We call this fibre bundle the Mostow bundle of $G / \Gamma$. The structure group is $N \Gamma / \Gamma_{0}$ as left translations where $\Gamma_{0}$ is the largest normal subgroup of $\Gamma$ which is normal in $N \Gamma$ (see $[17])$.

Let $\rho: G \rightarrow G L\left(V_{\rho}\right)$ be a representation such that the restriction $\rho_{\left.\right|_{N}}$ is a unipotent representation. For the Mostow bundle $p: G / \Gamma \rightarrow(G / N) /$ $(\Gamma / \Gamma \cap N)$, we define the vector bundle

$$
\mathbf{H}^{q}(N / \Gamma \cap N)=\sqcup_{x \in(G / N) /(\Gamma / \Gamma \cap N)} H^{q}\left(p^{-1}(x), E_{\rho}\right)
$$

over the torus $(G / N) /(\Gamma / \Gamma \cap N)$. By Theorem 1.1, we have $H^{q}\left(p^{-1}(x), E_{\rho}\right)$ $\cong H^{q}\left(\mathfrak{n}, V_{\rho}\right)$. Hence let $\Lambda_{q}: G / N \rightarrow G L\left(H^{q}\left(\mathfrak{n}, V_{\rho}\right)\right)$ be the representation induced by the extension $1 \rightarrow N \rightarrow G \rightarrow G / N \rightarrow 1$, then we can regard $\mathbf{H}^{q}(N / \Gamma \cap N)$ as the flat bundle $E_{\Lambda_{q}}$. We consider the filtration

$$
F^{p} \bigwedge_{p+q} \mathfrak{g}_{\mathbb{C}}^{*}=\left\{\omega \in \bigwedge^{p+q} \mathfrak{g}_{\mathbb{C}}^{*} \mid \omega\left(X_{1}, \ldots, X_{p+q}\right)=0 \text { for } X_{1}, \ldots, X_{p+1} \in \mathfrak{n}_{\mathbb{C}}\right\}
$$

This filtration gives the filtration of the cochain complex $\bigwedge \mathfrak{g}_{\mathbb{C}}^{*} \otimes V_{\rho}$ and the filtration of the de Rham complex $A^{*}\left(G / \Gamma, E_{\rho}\right)$. We consider the spectral sequence $E_{*}^{*, *}(\mathfrak{g})$ of $\Lambda \mathfrak{g}_{\mathbb{C}}^{*} \otimes V_{\rho}$ and the spectral sequence $E_{*}^{*, *}(G / \Gamma)$ of $A^{*}\left(G / \Gamma, E_{\rho}\right)$. Set $G / N=A$ and $\Gamma / \Gamma \cap N=\Delta$ and $\mathfrak{a}=\mathfrak{g} / \mathfrak{n}$. Then we have the commutative diagram

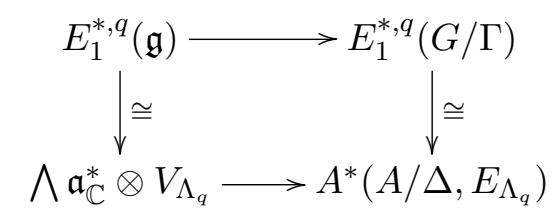

(see [6], [15, Section 7]). 


\subsection{Proof of Theorem $\mathbf{1 . 3}$}

Proof. Consider the spectral sequence $E_{*}^{*, *}(\mathfrak{g})$ of

$$
\bigoplus_{\alpha \in \mathcal{A}_{(G, N)}} \bigwedge \mathfrak{g}_{\mathbb{C}}^{*} \otimes V_{\alpha} \otimes V_{\rho}
$$

and the spectral sequence $E_{*}^{*, *}(G / \Gamma)$ of

$$
\bigoplus_{E_{\alpha} \in \mathcal{A}_{(G, N)}(\Gamma)} A^{*}\left(G / \Gamma, E_{\alpha} \otimes E_{\rho}\right) .
$$

Set $A=G / N$ and $\Delta=\Gamma / \Gamma \cap N$ and $\mathfrak{a}=\mathfrak{g} / \mathfrak{n}$. Since we can identify $\mathcal{A}_{(G, N)}$ $\left(\operatorname{resp} . \mathcal{A}_{(G, N)}(\Gamma)\right)$ with $\operatorname{Hom}\left(A, \mathbb{C}^{*}\right)(\operatorname{resp} . \mathcal{H}(A / \Delta))$, we have the commutative diagram

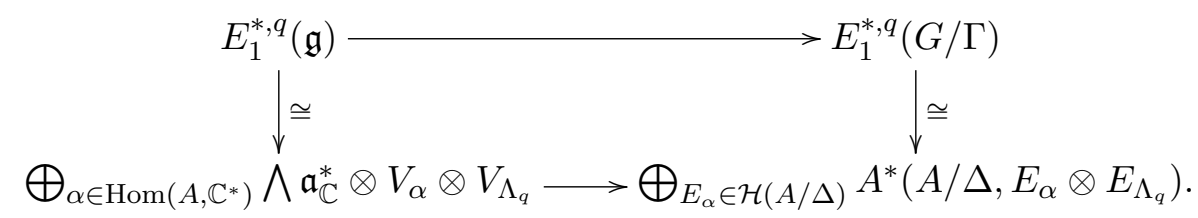

By Proposition 3.3, the homomorphism $E_{1}^{*, *}(\mathfrak{g}) \rightarrow E_{1}^{*, *}(G / \Gamma)$ induces a cohomology isomorphism and hence we have an isomorphism $E_{2}^{*, *}(\mathfrak{g}) \cong$ $E_{2}^{*, *}(G / \Gamma)$. Hence the theorem follows.

\section{Proof of Theorem 1.4}

\subsection{Dolbeault cohomology of tori}

First we prove Theorem 1.2 by Sakane's Theorem [16].

Proof of Theorem 1.2. In case $\operatorname{dim} V_{\sigma}=1, \sigma$ is trivial and the theorem follows from Sakane's Theorem [16].

In case $\operatorname{dim} V_{\sigma}=n>1$, since $\sigma$ is unipotent, we have a $(n-1)$ dimensional $G$-submodule $V_{\sigma^{\prime}} \subset V_{\sigma}$ such that $V_{\sigma} / V_{\sigma^{\prime}}$ is the trivial submodule. Then we have the spectral sequences

$$
0 \longrightarrow \bigwedge \mathfrak{g}^{*} \otimes V_{\sigma^{\prime}} \longrightarrow \bigwedge \mathfrak{g}^{*} \otimes V_{\sigma} \longrightarrow \bigwedge \mathfrak{g}^{*} \otimes V_{\sigma} / V_{\sigma^{\prime}} \longrightarrow 0
$$

and

$0 \longrightarrow A^{0, *}\left(G / \Gamma, L_{\bar{\sigma}^{\prime}}\right) \longrightarrow A^{0, *}\left(G / \Gamma, L_{\bar{\sigma}}\right) \longrightarrow A^{0, *}\left(G / \Gamma, L_{\bar{\sigma}} / L_{\bar{\sigma}^{\prime}}\right) \longrightarrow 0$. 
We have the commutative diagram

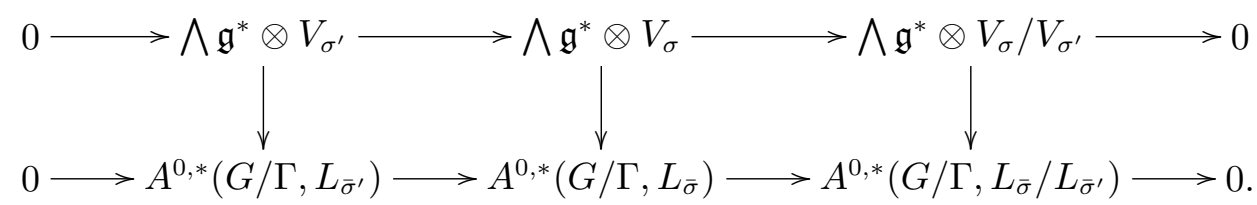

Considering the long exact sequence of cohomologies, by the five lemma, the theorem follows inductively.

Let $A$ be a simply connected complex abelian group with a lattice $\Gamma$ and $\mathfrak{a}$ the Lie algebra of $A$.

Lemma 4.1. Let $\sigma: A \rightarrow G L\left(V_{\sigma}\right)$ be a holomorphic representation. Suppose $\sigma=\beta \otimes \phi$ such that $\beta$ is a character of $A$ and $\phi$ is a unipotent representation. Then we have:

If $\beta$ is non-trivial, then we have

$$
H^{*}\left(\mathfrak{a}, V_{\sigma}\right)=0
$$

If the holomorphic line bundle $L_{\bar{\beta}}$ is non-trivial, then we have

$$
H^{0, *}\left(A / \Gamma, L_{\bar{\sigma}}\right)=0
$$

Proof. In case $\operatorname{dim} V_{\sigma}=1$, the lemma is proved in [10].

In case $\operatorname{dim} V_{\sigma}=n>1$, by the triangulation of $\sigma$, we have a $(n-1)$ dimensional $A$-submodule $V_{\sigma^{\prime}}$ such that $V_{\sigma} / V_{\sigma^{\prime}}=V_{\beta}$. Then we have the exact sequence

$$
0 \longrightarrow A^{0, *}\left(A / \Gamma, L_{\bar{\sigma}^{\prime}}\right) \longrightarrow A^{0, *}\left(A / \Gamma, L_{\bar{\sigma}}\right) \longrightarrow A^{0, *}\left(A / \Gamma, L_{\bar{\sigma}} / L_{\bar{\sigma}^{\prime}}\right) \longrightarrow 0 \text {. }
$$

Considering the long exact sequence of cohomologies, the lemma follows inductively.

By similar proof of Lemma 3.2, we have the following lemma.

Lemma 4.2. Let $\sigma: A \rightarrow G L\left(V_{\sigma}\right)$ be a holomorphic representation. Then we have a basis of $V_{\sigma}$ such that $\sigma$ is represented by

$$
\sigma=\bigoplus_{i=1}^{k} \alpha_{i} \otimes \phi_{i}
$$

for holomorphic characters $\alpha_{i}$ and holomorphic unipotent representations $\phi_{i}$. 
Let $\left\{V_{\alpha}\right\}_{\alpha \in \operatorname{Hom}_{\text {hol }}\left(A, \mathbb{C}^{*}\right)}$ be the set of all one-dimensional holomorphic representations of $A$ and $\mathcal{H}_{\text {hol }}(A / \Gamma)=\left\{L_{\bar{\alpha}}\right\}$ the set of all the isomorphism classes of holomorphic line bundles given by $\left\{V_{\alpha}\right\}_{\alpha \in \operatorname{Hom}_{\text {hol }}\left(A, \mathbb{C}^{*}\right)}$. We notice that the correspondence $\left\{V_{\alpha}\right\}_{\alpha \in \operatorname{Hom}_{\text {hol }}\left(A, \mathbb{C}^{*}\right)} \rightarrow \mathcal{H}(A / \Gamma)$ is not injective. We consider the direct sums

$$
\bigoplus_{\alpha \in \operatorname{Hom}_{\text {hol }}\left(A, \mathbb{C}^{*}\right)} \bigwedge \mathfrak{a}^{*} \otimes V_{\alpha} \otimes V_{\sigma}
$$

and

$$
\bigoplus_{L_{\bar{\alpha}} \in \mathcal{H}_{\mathrm{hol}}(A / \Gamma)} A^{0, *}\left(A / \Gamma, L_{\bar{\alpha}} \otimes L_{\bar{\sigma}}\right)
$$

Proposition 4.3. The inclusion

$$
\bigoplus_{\alpha \in \operatorname{Hom}_{\mathrm{hol}}\left(A, \mathbb{C}^{*}\right)} \bigwedge \mathfrak{a}^{*} \otimes V_{\alpha} \otimes V_{\sigma} \rightarrow \bigoplus_{L_{\bar{\alpha}} \in \mathcal{H}_{\mathrm{hol}}(A / \Gamma)} A^{0, *}\left(A / \Gamma, L_{\bar{\alpha}} \otimes L_{\bar{\sigma}}\right)
$$

induces a cohomology isomorphism.

Proof. Using Theorem 1.2 and Lemmas 4.1 and 4.2, we can prove the proposition by similar argument of the proof of Proposition 3.3

\subsection{Mostow bundle and spectral sequence}

Let $G$ be a simply connected complex solvable Lie group with a lattice $\Gamma$ and $\mathfrak{g}$ be the Lie algebra of $G$. Then the Mostow bundle

$$
N / \Gamma \cap N=N \Gamma / \Gamma \longrightarrow G / \Gamma \longrightarrow G / N \Gamma=(G / N) /(\Gamma / \Gamma \cap N)
$$

is holomorphic.

Let $\sigma: G \rightarrow G L\left(V_{\sigma}\right)$ be a representation such that the restriction $\sigma_{\left.\right|_{N}}$ is a unipotent representation. For the Mostow bundle $p: G / \Gamma \rightarrow(G / N) /(\Gamma / \Gamma \cap$ $N$ ), we define the vector bundle

$$
\mathbf{H}^{0, q}(N / \Gamma \cap N)=\sqcup_{x \in(G / N) /(\Gamma / \Gamma \cap N)} H^{0, q}\left(p^{-1}(x), L_{\bar{\sigma}}\right)
$$

over the torus $(G / N) /(\Gamma / \Gamma \cap N)$. By Theorem 1.2 , we have $H^{0, q}\left(p^{-1}(x), L_{\bar{\sigma}}\right)$ $\cong H^{q}\left(\mathfrak{n}, V_{\sigma}\right)$. Hence let $\Lambda_{q}: G / N \rightarrow G L\left(H^{q}\left(\mathfrak{n}, V_{\sigma}\right)\right)$ be the representation induced by the extension $1 \rightarrow N \rightarrow G \rightarrow G / N \rightarrow 1$, then we can regard 
$\mathbf{H}^{0, q}(N / \Gamma \cap N)$ as the flat holomorphic bundle $L_{\bar{\Lambda}}$. We consider the filtration

$$
F^{p} \bigwedge_{p+q} \mathfrak{g}^{*}=\left\{\omega \in \bigwedge^{p+q} \mathfrak{g}^{*} \mid \omega\left(X_{1}, \ldots, X_{p+q}\right)=0 \text { for } X_{1}, \ldots, X_{p+1} \in \mathfrak{n}\right\} .
$$

This filtration gives the filtration of the cochain complex $\bigwedge \mathfrak{g}^{*} \otimes V_{\sigma}$ and the filtration of the Dolbeault complex $A^{0, *}\left(G / \Gamma, L_{\bar{\sigma}}\right)=C^{\infty}\left(G / \Gamma, L_{\bar{\sigma}}\right) \otimes \wedge \mathfrak{g}^{*}$. We consider the spectral sequence ${ }_{\text {Dol }} E_{*}^{*, *}(\mathfrak{g})$ of $\bigwedge \mathfrak{g}^{*} \otimes V_{\sigma}$ and the spectral sequence $\operatorname{Dol}_{*}^{* * *}(G / \Gamma)$ of $A^{0, *}\left(G / \Gamma, L_{\bar{\sigma}}\right)$. Set $G / N=A$ and $\Gamma / \Gamma \cap N=\Delta$ and $\mathfrak{a}=\mathfrak{g} / \mathfrak{n}$. By Borel's result [7, Appendix 2], we have the commutative diagram

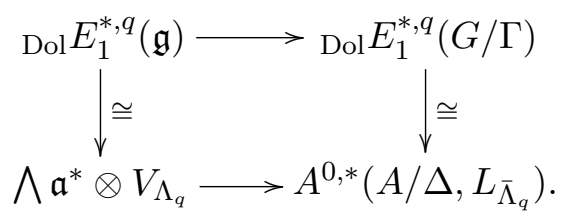

\subsection{Proof of theorem}

Proof. Consider the spectral sequence ${ }_{\text {Dol }} E_{*}^{*, *}(\mathfrak{g})$ of

$$
\bigoplus_{\alpha \in \mathcal{B}_{(G, N)}} \bigwedge \mathfrak{g}^{*} \otimes V_{\alpha} \otimes V_{\sigma}
$$

and the spectral sequence ${ }_{\operatorname{Dol}} E_{*}^{*, *}(G / \Gamma)$ of

$$
\bigoplus_{L_{\bar{\alpha}} \in \mathcal{B}_{(G, N)}(\Gamma)} A^{0, *}\left(G / \Gamma, L_{\bar{\alpha}} \otimes L_{\bar{\sigma}}\right) .
$$

Set $A=G / N$ and $\Delta=\Gamma / \Gamma \cap N$ and $\mathfrak{a}=\mathfrak{g} / \mathfrak{n}$. Since we can identify $\mathcal{B}_{(G, N)}$ (resp. $\left.\mathcal{B}_{(G, N)}(\Gamma)\right)$ with $\operatorname{Hom}_{\text {hol }}\left(A, \mathbb{C}^{*}\right)\left(\right.$ resp. $\mathcal{H}_{\text {hol }}(A / \Delta)$ as Section 4.1$)$, we have the commutative diagram

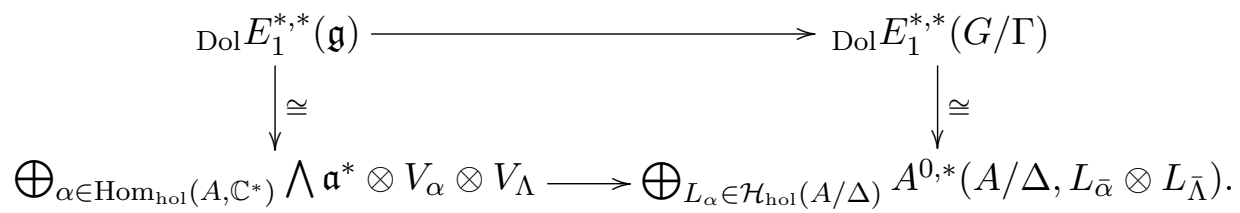

By Proposition 4.3, the homomorphism $\operatorname{Dol}_{1}^{* * *}(\mathfrak{g}) \rightarrow{ }_{\operatorname{Dol}} E_{1}^{*, *}(G / \Gamma)$ induces a cohomology isomorphism and hence we have an isomorphism $\operatorname{Dol}_{2}^{*, *}(\mathfrak{g}) \cong$ ${ }_{\operatorname{Dol}} E_{2}^{*, *}(G / \Gamma)$. Hence the theorem follows. 


\section{Construction of finite cochain complex (de Rham case)}

We will use the following proposition.

Proposition 5.1 ([2, Proposition 3.3]). Let $G$ be a simply connected solvable Lie group $G$ and $N$ the nilradical of $G$. Then we have a simply connected nilpotent subgroup $C \subset G$ such that $G=C \cdot N$.

Remark 2. This proposition is given by the decomposition (not necessarily direct sum) $\mathfrak{g}=\mathfrak{c}+\mathfrak{n}$ (see [3, Theorem 2.2]). Since this decomposition is compatible with any field (see [3, Theorem 2.2]), if $G$ is complex Lie group we can take a subgroup $C$ also complex.

Let $G$ be a simply connected solvable Lie group and $\mathfrak{g}$ be the Lie algebra of $G$. Let $N$ be the nilradical of $G$. Let $\rho: G \rightarrow G L\left(V_{\rho}\right)$ be a representation. Suppose the restriction $\rho_{\left.\right|_{N}}$ is unipotent. We consider the direct sum

$$
\bigoplus_{\alpha \in \mathcal{A}_{(G, N)}} \bigwedge \mathfrak{g}_{\mathbb{C}}^{*} \otimes V_{\alpha} \otimes V_{\rho}
$$

Then we have the $G$-action on this cochain complex via $\bigoplus \operatorname{Ad} \otimes \alpha \otimes \rho$. Since this action is extension of the Lie derivation, the induced action on the cohomology is trivial. Consider the semi-simple part

$$
\left(\left(\bigoplus_{\alpha \in \mathcal{A}_{(G, N)}} \operatorname{Ad} \otimes \alpha \otimes \rho\right)(g)\right)_{s}=\bigoplus_{\alpha \in \mathcal{A}_{(G, N)}}\left(\operatorname{Ad}_{g}\right)_{s} \otimes \alpha(g) \otimes(\rho(g))_{s}
$$

Take a simply connected nilpotent subgroup $C \subset G$ as Proposition 5.1. Since $C$ is nilpotent, the map

$$
\begin{aligned}
\Phi: C \ni c & \mapsto \bigoplus_{\alpha \in \mathcal{A}_{(G, N)}}\left(\operatorname{Ad}_{g}\right)_{s} \otimes \alpha(g) \otimes(\rho(g))_{s} \\
& \in \operatorname{Aut}\left(\bigoplus_{\alpha \in \mathcal{A}_{(G, N)}} \bigwedge \mathfrak{g}_{\mathbb{C}}^{*} \otimes V_{\alpha} \otimes V_{\rho}\right)
\end{aligned}
$$

is a homomorphism. We denote by

$$
\left(\bigoplus_{\alpha \in \mathcal{A}_{(G, N)}} \bigwedge \mathfrak{g}_{\mathbb{C}}^{*} \otimes V_{\alpha} \otimes V_{\rho}\right)^{\Phi(C)}
$$


the subcomplex consisting of the $\Phi(C)$-invariant elements.

Lemma 5.2. The inclusion

$$
\left(\bigoplus_{\alpha \in \mathcal{A}_{(G, N)}} \bigwedge \mathfrak{g}_{\mathbb{C}}^{*} \otimes V_{\alpha} \otimes V_{\rho}\right)^{\Phi(C)} \subset \bigoplus_{\alpha \in \mathcal{A}_{(G, N)}} \bigwedge \mathfrak{g}_{\mathbb{C}}^{*} \otimes V_{\alpha} \otimes V_{\rho}
$$

induces a cohomology isomorphism.

Proof. Since the induced $G$-action on the cohomology $H^{*}\left(\bigoplus_{\alpha \in \mathcal{A}_{(G, N)}} \wedge \mathfrak{g}_{\mathbb{C}}^{*} \otimes\right.$ $\left.V_{\alpha} \otimes V_{\rho}\right)$ is trivial and $\Phi(C)$-action is semi-simple part of $G$-action, the induced $\Phi(C)$-action on the cohomology $H^{*}\left(\bigoplus_{\alpha \in \mathcal{A}_{(G, N)}} \wedge \mathfrak{g}_{\mathbb{C}}^{*} \otimes V_{\alpha} \otimes V_{\rho}\right)$ is also trivial and hence

$$
H^{*}\left(\bigoplus_{\alpha \in \mathcal{A}_{(G, N)}} \bigwedge \mathfrak{g}_{\mathbb{C}}^{*} \otimes V_{\alpha} \otimes V_{\rho}\right)^{\Phi(C)}=H^{*}\left(\bigoplus_{\alpha \in \mathcal{A}_{(G, N)}} \bigwedge \mathfrak{g}_{\mathbb{C}}^{*} \otimes V_{\alpha} \otimes V_{\rho}\right)
$$

Since $\Phi$ is diagonalizable, we have

$$
\begin{aligned}
& H^{*}\left(\left(\bigoplus_{\alpha \in \mathcal{A}_{(G, N)}} \bigwedge \mathfrak{g}_{\mathbb{C}}^{*} \otimes V_{\alpha} \otimes V_{\rho}\right)^{\Phi(C)}\right) \\
& =H^{*}\left(\bigoplus_{\alpha \in \mathcal{A}_{(G, N)}} \bigwedge \mathfrak{g}_{\mathbb{C}}^{*} \otimes V_{\alpha} \otimes V_{\rho}\right)^{\Phi(C)} .
\end{aligned}
$$

Hence the lemma follows.

The subcomplex $\left(\bigoplus_{\alpha \in \mathcal{A}_{(G, N)}} \wedge \mathfrak{g}_{\mathbb{C}}^{*} \otimes V_{\alpha} \otimes V_{\rho}\right)^{\Phi(C)}$ is desired subcomplex $A^{*}$ as in Theorem 1.5. Using certain basis, we see that this complex is finitedimensional and write down the subcomplex $A_{\Gamma}^{*}$ as Corollary 1.6 explicitly.

We have a basis $X_{1}, \ldots, X_{n}$ of $\mathfrak{g}_{\mathbb{C}}$ such that $\left(\operatorname{Ad}_{c}\right)_{s}=\operatorname{diag}\left(\alpha_{1}(c), \ldots\right.$, $\left.\alpha_{n}(c)\right)$ for $c \in C$. Let $x_{1}, \ldots, x_{n}$ be the basis of $\mathfrak{g}_{\mathbb{C}}^{*}$ which is dual to $X_{1}, \ldots$, $X_{n}$. We have a basis $v_{1}, \ldots, v_{m}$ of $V_{\rho}$ such that $(\rho(c))_{s}=\operatorname{diag}\left(\alpha_{1}^{\prime}(c), \ldots\right.$, $\left.\alpha_{m}^{\prime}(c)\right)$ for any $c \in C$. Let $v_{\alpha}$ be a basis of $V_{\alpha}$ for each character $\alpha \in \mathcal{A}_{(G, N)}$. By $G=C \cdot N$, we have $G / N=C / C \cap N$ and hence we have $\mathcal{A}_{(G, N)}$ $=\mathcal{A}_{C, C \cap N}=\left\{\alpha \in \operatorname{Hom}\left(C, \mathbb{C}^{*}\right) \mid \alpha_{\left.\right|_{C \cap N}}=1\right\}$. 
For a multi-index $I=\left\{i_{1}, \ldots, i_{p}\right\}$ we write $x_{I}=x_{i_{1}} \wedge \cdots \wedge x_{i_{p}}$, and $\alpha_{I}=$ $\alpha_{i_{1}} \cdots \alpha_{i_{p}}$. We consider the basis

$$
\left\{x_{I} \otimes v_{\alpha} \otimes v_{k}\right\}_{I \subset\{1, \ldots, n\}, \alpha \in \mathcal{A}_{C, C \cap N}, k \in\{1, \ldots, m\}}
$$

of $\bigoplus_{\alpha \in \mathcal{A}_{C, C \cap N}} \wedge \mathfrak{g}_{\mathbb{C}}^{*} \otimes V_{\alpha} \otimes V_{\rho}$. Since the action

$$
\Phi: C \rightarrow \operatorname{Aut}\left(\bigoplus_{\alpha} \bigwedge \mathfrak{g}^{*} \otimes V_{\alpha} \otimes V_{\rho}\right)
$$

is the semi-simple part of $(\bigoplus \operatorname{Ad} \otimes \alpha \otimes \rho)_{\left.\right|_{C}}$, we have

$$
\Phi(a)\left(x_{I} \otimes v_{\alpha} \otimes v_{k}\right)=\alpha_{I}^{-1} \alpha \alpha_{k}^{\prime} x_{I} \otimes v_{\alpha} \otimes v_{k} .
$$

Hence we have

$$
\begin{aligned}
\left(\bigoplus_{\alpha} \bigwedge \mathfrak{g}_{\mathbb{C}}^{*} \otimes V_{\alpha} \otimes V_{\rho}\right)^{\Phi(C)}= & \left\langle x_{I} \otimes v_{\alpha_{I} \alpha_{k}^{\prime-1}} \otimes v_{k} \otimes\right\rangle_{I \subset\{1, \ldots, n\}, k \in\{1, \ldots, m\}} \\
= & \bigwedge\left\langle x_{1} \otimes v_{\alpha_{1}}, \ldots, x_{n} \otimes v_{\alpha_{n}}\right\rangle \\
& \otimes\left\langle v_{\alpha_{1}^{\prime-1}} \otimes v_{1}, \ldots, v_{\alpha_{m}^{\prime-1}} \otimes v_{m}\right\rangle .
\end{aligned}
$$

Finally we construct a finite-dimensional complex $A_{\Gamma}^{*}$ which computes the de Rham cohomology $H^{*}\left(G / \Gamma, E_{\rho}\right)$.

Corollary 5.3. Let $A_{\Gamma}^{*}$ be the subcomplex of $\left(\bigoplus_{\alpha} \wedge \mathfrak{g}_{\mathbb{C}}^{*} \otimes V_{\alpha} \otimes V_{\rho}\right)^{\Phi(C)}$ defined as

$$
A_{\Gamma}^{*}=\left\langle x_{I} \otimes v_{\alpha_{I} \alpha_{k}^{\prime-1}} \otimes v_{k} \mid\left(\alpha_{I} \alpha_{k}^{\prime-1}\right)_{\left.\right|_{\Gamma}}=1\right\rangle .
$$

Then we have an isomorphism

$$
H^{*}\left(A_{\Gamma}^{*}\right) \cong H^{*}\left(G / \Gamma, E_{\rho}\right)
$$

Proof. Consider the inclusion

$$
\iota:\left(\bigoplus_{\alpha \in \mathcal{A}_{G, N}} \bigwedge \mathfrak{g}_{\mathbb{C}}^{*} \otimes V_{\alpha} \otimes V_{\rho}\right)^{\Phi(C)} \rightarrow \bigoplus_{E_{\alpha} \in \mathcal{A}_{(G, N)}(\Gamma)} A^{*}\left(G / \Gamma, E_{\alpha} \otimes E_{\rho}\right) .
$$

$\iota\left(x_{I} \otimes v_{\alpha_{I} \alpha_{k}^{\prime-1}} \otimes v_{k}\right) \in A^{*}\left(G / \Gamma, E_{\rho}\right)$ if and only if $\left(\alpha_{I} \alpha_{k}^{\prime-1} \rho\right)_{\left.\right|_{\Gamma}}=\rho_{\left.\right|_{\Gamma}}$. Hence we have $\iota^{-1}\left(A^{*}\left(G / \Gamma, E_{\rho}\right)\right)=A_{\Gamma}^{*}$. 
Corollary 5.4. We consider the following conditions:

$\left(\diamond_{1}\right)$ For each multi-index $I=\left\{i_{1}, \ldots, i_{p}\right\}$ and $k \in\{1, \ldots, m\}$, the character $\alpha_{I} \alpha_{k}^{\prime-1}$ is trivial if and only if the restriction $\left(\alpha_{I} \alpha_{k}^{\prime-1}\right)_{\left.\right|_{\Gamma}}$ is trivial.

$\left(\triangle_{2}\right)$ For each multi-index $I=\left\{i_{1}, \ldots, i_{p}\right\}$ and $k \in\{1, \ldots, m\}$, the character $\alpha_{I} \alpha_{k}^{\prime-1}$ is trivial or non-unitary.

If the condition $\left(\diamond_{1}\right)$ or $\left(\diamond_{2}\right)$ holds, then we have an isomorphism

$$
H^{*}\left(\mathfrak{g}, V_{\rho}\right) \cong H^{*}\left(G / \Gamma, E_{\rho}\right)
$$

Proof. If the condition $\left(\diamond_{1}\right)$ holds, then we have $A_{\Gamma}^{*}=\left(\bigwedge \mathfrak{g}_{\mathbb{C}}^{*} \otimes V_{\rho}\right)^{\Phi(C)}$. Hence we have

$$
H^{*}\left(G / \Gamma, E_{\rho}\right) \cong H\left(\bigwedge \mathfrak{g}_{\mathbb{C}}^{*} \otimes V_{\rho}\right)^{\Phi(C)} \cong H^{*}\left(\mathfrak{g}, V_{\rho}\right) .
$$

The condition $\left(\diamond_{2}\right)$ is special case of the condition $\left(\diamond_{1}\right)$. Hence the corollary follows.

Remark 3. For a representation $\rho: G \rightarrow G L\left(V_{\rho}\right)$ such that the restriction $\rho_{\left.\right|_{N}}$ is trivial, the condition (M) (resp. $\left.(\mathrm{H})\right)$ in Section 1 is a special case of the condition $\left(\diamond_{1}\right)$ (resp. $\left.\left(\diamond_{2}\right)\right)$

Remark 4. Let $\mathfrak{c}$ be the Lie algebra of $C$. Take a subvector $V \subset \mathfrak{c}$ (not necessarily Lie algebra) such that $\mathfrak{g}=V \oplus \mathfrak{n}$. Then we define the map

$$
\operatorname{ad}_{s}: \mathfrak{g}=V \oplus \mathfrak{n} \ni A+X \mapsto\left(\operatorname{ad}_{A}\right)_{s} \in D(\mathfrak{g}),
$$

where $\left(\operatorname{ad}_{A}\right)_{S}$ is the semi-simple part of $\operatorname{ad}_{A}$ and $D(\mathfrak{g})$ is the Lie algebra of derivations of $\mathfrak{g}$. This map is a Lie algebra homomorphism and a diagonalizable representation (see $[4,9]$ ). Let $\operatorname{Ad}_{s}: G \rightarrow \operatorname{Aut}(\mathfrak{g})$ be the extension of $\operatorname{ad}_{s}$. Then this map is identified with the map

$$
G=C \cdot N \ni c \cdot n \mapsto\left(\operatorname{Ad}_{c}\right) \in \operatorname{Aut}(\mathfrak{g}) .
$$

We define the Lie algebra $\mathfrak{u}_{G} \subset D(\mathfrak{g}) \ltimes \mathfrak{g}$ as

$$
\mathfrak{u}_{G}=\left\{X-\operatorname{ad}_{s X} \mid X \in \mathfrak{g}\right\} .
$$

Consider the above basis $\left\{x_{1}, \ldots, x_{n}\right\}$ of $\mathfrak{g}_{\mathbb{C}}^{*}$. Then in [9] the author showed that we have an isomorphism

$$
\bigwedge\left\langle x_{1} \otimes v_{\alpha_{1}}, \ldots, x_{n} \otimes v_{\alpha_{n}}\right\rangle \cong \bigwedge\left(\mathfrak{u}_{G} \otimes \mathbb{C}\right)^{*} .
$$


(This fact gives the new developments of de Rham homotopy theory on solvmanifolds. See [9].) Hence we can regard

$$
\begin{aligned}
\left(\bigoplus_{\alpha \in \mathcal{A}_{G, N}} \bigwedge \mathfrak{g}_{\mathbb{C}}^{*} \otimes V_{\alpha} \otimes V_{\rho}\right)^{\Phi(C)}= & \bigwedge\left\langle x_{1} \otimes v_{\alpha_{1}}, \ldots, x_{n} \otimes v_{\alpha_{n}}\right\rangle \\
& \otimes\left\langle v_{\alpha_{1}^{\prime-1}} \otimes v_{1}, \ldots, v_{\alpha_{m}^{\prime-1}} \otimes v_{m}\right\rangle
\end{aligned}
$$

as the cochain complex of the nilpotent Lie algebra $\mathfrak{u}_{G}$ with values in some representation.

\section{Construction of finite cochain complex (Dolbeault case)}

In this case, we can say almost same argument for the de Rham case without difficulties. Let $G$ be a simply connected solvable Lie group and $\mathfrak{g}$ be the Lie algebra of $G$. Let $N$ be the nilradical of $G$. Let $\sigma: G \rightarrow G L\left(V_{\sigma}\right)$ be a holomorphic representation. Suppose the restriction $\sigma_{\left.\right|_{N}}$ is unipotent. We consider the direct sum

$$
\bigoplus_{\alpha \in \mathcal{B}_{(G, N)}} \bigwedge \mathfrak{g}^{*} \otimes V_{\alpha} \otimes V_{\rho}
$$

Then we have the $G$-action on this cochain complex via $\bigoplus \operatorname{Ad} \otimes \alpha \otimes \rho$. Consider the semi-simple part

$$
\left(\left(\bigoplus_{\alpha \in \mathcal{B}_{(G, N)}} \operatorname{Ad} \otimes \alpha \otimes \rho\right)(g)\right)_{s}=\bigoplus_{\alpha \in \mathcal{B}_{(G, N)}}\left(\operatorname{Ad}_{g}\right)_{s} \otimes \alpha(g) \otimes(\rho(g))_{s}
$$

Take a simply connected complex nilpotent subgroup $C \subset G$ as Proposition 5.1 and Remark 2. Since $C$ is nilpotent, the map

$$
\begin{aligned}
\Phi: C \ni c & \mapsto \bigoplus_{\alpha \in \mathcal{B}_{(G, N)}}\left(\operatorname{Ad}_{g}\right)_{s} \otimes \alpha(g) \otimes(\rho(g))_{s} \\
& \in \operatorname{Aut}\left(\bigoplus_{\alpha \in \mathcal{B}_{(G, N)}} \bigwedge \mathfrak{g}^{*} \otimes V_{\alpha} \otimes V_{\sigma}\right)
\end{aligned}
$$


is a homomorphism. We denote by

$$
\left(\bigoplus_{\alpha \in \mathcal{A}_{(G, N)}} \bigwedge \mathfrak{g}^{*} \otimes V_{\alpha} \otimes V_{\sigma}\right)^{\Phi(C)}
$$

the subcomplex consisting of the $\Phi(C)$-invariant elements. By similar proof of Lemma 5.2, we have:

Lemma 6.1. The inclusion

$$
\left(\bigoplus_{\alpha \in \mathcal{B}_{(G, N)}} \bigwedge \mathfrak{g}^{*} \otimes V_{\alpha} \otimes V_{\sigma}\right)^{\Phi(C)} \subset \bigoplus_{\alpha \in \mathcal{B}_{(G, N)}} \bigwedge \mathfrak{g}^{*} \otimes V_{\alpha} \otimes V_{\sigma}
$$

induces a cohomology isomorphism.

We have a basis $X_{1}, \ldots, X_{n}$ of $\mathfrak{g}$ such that $\left(\operatorname{Ad}_{c}\right)_{s}=\operatorname{diag}\left(\alpha_{1}(c), \ldots\right.$, $\left.\alpha_{n}(c)\right)$ for $c \in C$. Let $x_{1}, \ldots, x_{n}$ be the basis of $\mathfrak{g}^{*}$ which is dual to $X_{1}, \ldots, X_{n}$. We have a basis $v_{1}, \ldots, v_{m}$ of $V_{\sigma}$ such that $(\sigma(c))_{s}=\operatorname{diag}\left(\alpha_{1}^{\prime}(c), \ldots, \alpha_{m}^{\prime}(c)\right)$ for any $c \in C$. Let $v_{\alpha}$ be a basis of $V_{\alpha}$ for each character $\alpha \in \mathcal{B}_{(G, N)}$. By $G=$ $C \cdot N$, we have $G / N=C / C \cap N$ and hence we have $\mathcal{B}_{(G, N)}=\mathcal{B}_{C, C \cap N}=$ $\left\{\alpha \in \operatorname{Hom}_{\text {hol }}\left(C, \mathbb{C}^{*}\right) \mid \alpha_{\left.\right|_{C \cap N}}=1\right\}$.

For a multi-index $I=\left\{i_{1}, \ldots, i_{p}\right\}$ we write $x_{I}=x_{i_{1}} \wedge \cdots \wedge x_{i_{p}}$, and $\alpha_{I}=$ $\alpha_{i_{1}} \cdots \alpha_{i_{p}}$. We consider the basis

$$
\left\{x_{I} \otimes v_{\alpha} \otimes v_{k}\right\}_{I \subset\{1, \ldots, n\}, \alpha \in \mathcal{A}_{C, C \cap N}, k \in\{1, \ldots, m\}}
$$

of $\bigoplus_{\alpha \in \mathcal{B}_{C, C \cap N}} \wedge \mathfrak{g}^{*} \otimes V_{\alpha} \otimes V_{\sigma}$. Since the action

$$
\Phi: C \rightarrow \operatorname{Aut}\left(\bigoplus_{\alpha} \bigwedge \mathfrak{g}^{*} \otimes V_{\alpha} \otimes V_{\sigma}\right)
$$

is the semi-simple part of $(\bigoplus \mathrm{Ad} \otimes \alpha \otimes \sigma)_{\left.\right|_{C}}$, we have

$$
\Phi(a)\left(x_{I} \otimes v_{\alpha} \otimes v_{k}\right)=\alpha_{I}^{-1} \alpha \alpha_{k}^{\prime} x_{I} \otimes v_{\alpha} \otimes v_{k}
$$


Hence we have

$$
\begin{aligned}
\left(\bigoplus_{\alpha} \bigwedge \mathfrak{g}^{*} \otimes V_{\alpha} \otimes V_{\sigma}\right)^{\Phi(C)}= & \left\langle x_{I} \otimes v_{\alpha_{I} \alpha_{k}^{\prime-1}} \otimes v_{k}\right\rangle_{I \subset\{1, \ldots, n\}, k \in\{1, \ldots, m\}} \\
= & \bigwedge\left\langle x_{1} \otimes v_{\alpha_{1}}, \ldots, x_{n} \otimes v_{\alpha_{n}}\right\rangle \\
& \otimes\left\langle v_{\alpha_{1}^{\prime-1}} \otimes v_{1}, \ldots, v_{\alpha_{m}^{\prime-1}} \otimes v_{m}\right\rangle .
\end{aligned}
$$

Corollary 6.2. Let $B_{\Gamma}^{*}$ be the subcomplex of $\left\langle x_{I} \otimes v_{\alpha_{I} \alpha_{k}^{\prime-1}} \otimes\right.$ $\left.v_{k}\right\rangle_{I \subset\{1, \ldots, n\}, k \in\{1, \ldots, m\}}$ defined as

$$
B_{\Gamma}^{*}=\left\langle x_{I} \otimes v_{\alpha_{I} \alpha_{k}^{\prime-1}} \otimes v_{k} \mid\left(\frac{\bar{\alpha}_{I} \bar{\alpha}_{k}^{\prime-1}}{\alpha_{I} \alpha_{k}^{\prime-1}}\right)_{\left.\right|_{\Gamma}}=1\right\rangle .
$$

Then we have an isomorphism

$$
H^{*}\left(B_{\Gamma}^{*}\right) \cong H^{0, *}\left(G / \Gamma, L_{\bar{\sigma}}\right) .
$$

Proof. It is known that we have the $1-1$ correspondence between the isomorphism classes of flat holomorphic line bundles over a complex torus and the unitary characters of its lattice (see [14]). By this, for $\alpha \in \mathcal{B}_{(G, N)}$, considering the unitary character $\frac{\bar{\alpha}}{\alpha}$, the holomorphic line bundle $L_{\bar{\alpha}}$ is trivial if and only if the restriction $\left(\frac{\bar{\alpha}}{\alpha}\right)_{\left.\right|_{\Gamma}}$ is trivial. Hence

$$
\iota\left(x_{I} \otimes v_{\alpha_{I} \alpha_{k}^{\prime-1}} \otimes v_{k}\right) \in A^{*}\left(G / \Gamma, L_{\bar{\sigma}}\right)
$$

if and only if the restriction $\left(\frac{\bar{\alpha}_{I} \bar{\alpha}_{k}^{\prime-1}}{\alpha_{I} \alpha_{k}^{\prime-1}}\right)_{\left.\right|_{\Gamma}}$ is trivial. Then we have $\iota^{-1}\left(A^{*}(G / \Gamma\right.$, $\left.\left.L_{\bar{\sigma}}\right)\right)=B_{\Gamma}^{*}$.

Corollary 6.3. We consider the following condition:

(*) For each multi-index $I=\left\{i_{1}, \ldots, i_{p}\right\}$ and $k \in\{1, \ldots, m\}$, the character $\alpha_{I} \alpha_{k}^{\prime-1}$ is trivial if and only if the restriction $\left(\frac{\bar{\alpha}_{I} \bar{\alpha}_{k}^{\prime-1}}{\alpha_{I} \alpha_{k}^{\prime-1}}\right)_{\left.\right|_{\Gamma}}$ is trivial.

If the condition $(\star)$ holds, then we have an isomorphism

$$
H^{*}\left(\mathfrak{g}, V_{\sigma}\right) \cong H^{0, *}\left(G / \Gamma, L_{\bar{\sigma}}\right) .
$$

Proof. Suppose the condition $(\star)$ holds. Then we have $B_{\Gamma}^{*}=\left(\bigwedge \mathfrak{g}^{*} \otimes V_{\sigma}\right)^{\Phi(C)}$. Hence we have

$$
H^{0, *}\left(G / \Gamma, L_{\bar{\sigma}}\right) \cong H^{*}\left(\bigwedge \mathfrak{g}^{*} \otimes V_{\sigma}\right)^{\Phi(C)} \cong H^{*}\left(\mathfrak{g}, V_{\sigma}\right)
$$


Remark 5. We define the nilpotent Lie algebra $\mathfrak{u}_{G}$ as Remark 4. In the complex case, $\mathfrak{u}_{G}$ is also a complex Lie algebra. As similar to Remark 4, we have

$$
\left(\bigoplus_{\alpha} \bigwedge \mathfrak{g}^{*} \otimes V_{\alpha}\right)^{\Phi(C)}=\bigwedge\left\langle x_{1} \otimes v_{\alpha_{1}}, \ldots, x_{n} \otimes v_{\alpha_{n}}\right\rangle \cong \bigwedge \mathfrak{u}_{G}^{*} .
$$

Suppose $G$ has a lattice $\Gamma$. We consider the cochain complex

$$
B_{\Gamma}^{*}=\left\langle x_{I} \otimes v_{\alpha_{I}} \mid\left(\frac{\bar{\alpha}_{I}}{\alpha_{I}}\right)_{\left.\right|_{\Gamma}}=1\right\rangle .
$$

Then we have an isomorphism $H^{0, *}\left(B_{\Gamma}^{*}\right) \cong H^{0, *}(G / \Gamma)$ by Corollary 6.2. We consider the following condition.

( $\square)$ For each $1 \leq i \leq n$, the restriction $\left(\frac{\bar{\alpha}_{i}}{\alpha_{i}}\right)_{\Gamma}$ is trivial.

If the condition ( $\square$ ) holds, then we have

$$
B_{\Gamma}^{*}=\bigwedge\left\langle x_{1} \otimes v_{\alpha_{1}}, \ldots, x_{n} \otimes v_{\alpha_{n}}\right\rangle \cong \bigwedge \mathfrak{u}_{G}^{*} .
$$

Let $U_{G}$ be the simply connected complex Lie group with the Lie algebra $\mathfrak{u}_{G}$. Then $U_{G}$ is the nilradical of the semi-simple splitting of $G$ (see [2]). It is known that if $G$ has a lattice, then $U_{G}$ has a lattice $\Gamma^{\prime}$ (see [1]).

Hence we have:

Corollary 6.4. Let $G$ be a simply connected complex solvable Lie group with a lattice $\Gamma$. If the condition ( $\square$ ) holds, then there exists a complex parallelizable nilmanifold $U_{G} / \Gamma^{\prime}$ such that we have an isomorphism

$$
H^{*, *}(G / \Gamma) \cong H^{*, *}\left(U_{G} / \Gamma^{\prime}\right) .
$$

By this corollary we have some solvmanifolds whose Dolbeault cohomology is isomorphic to the Dolbeault cohomology of nilmanifolds.

\section{Example}

Let $G=\mathbb{C} \ltimes_{\phi} \mathbb{C}^{2}$ such that

$$
\phi(z)=\left(\begin{array}{cc}
e^{z} & 0 \\
0 & e^{-z}
\end{array}\right) .
$$


Then we have $a+\sqrt{-1} b, c+\sqrt{-1} d \in \mathbb{C}$ such that $\mathbb{Z}(a+\sqrt{-1} b)+\mathbb{Z}(c+$ $\sqrt{-1} d)$ is a lattice in $\mathbb{C}$ and $\phi(a+\sqrt{-1} b)$ and $\phi(c+\sqrt{-1} d)$ are conjugate to elements of $S L(4, \mathbb{Z})$ where we regard $S L(2, \mathbb{C}) \subset S L(4, \mathbb{R})$ (see [5]). Hence we have a lattice $\Gamma=(\mathbb{Z}(a+\sqrt{-1} b)+\mathbb{Z}(c+\sqrt{-1} d)) \ltimes_{\phi} \Gamma^{\prime \prime}$ such that $\Gamma^{\prime \prime}$ is a lattice of $\mathbb{C}^{2}$.

\subsection{Twisted de Rham cohomology $H^{1}\left(G / \Gamma, E_{\mathrm{Ad}}\right)$}

For a coordinate $\left(w, z_{1}, z_{2}\right) \in \mathbb{C} \ltimes_{\phi} \mathbb{C}^{2}$ we have the basis $\left\{v_{1}, \ldots, v_{6}\right\}$ of $\mathfrak{g}_{\mathbb{C}}$ such that

$$
\begin{aligned}
& v_{1}=e^{w} \frac{\partial}{\partial z_{1}}, \quad v_{2}=e^{\bar{w}} \frac{\partial}{\partial \bar{z}_{1}}, \quad v_{3}=e^{-w} \frac{\partial}{\partial z_{2}}, \quad v_{4}=e^{-\bar{w}} \frac{\partial}{\partial \bar{z}_{2}} \\
& v_{5}=\frac{\partial}{\partial w}, \quad v_{6}=\frac{\partial}{\partial \bar{w}}
\end{aligned}
$$

Consider the dual basis

$$
e^{-w} d z_{1}, e^{-\bar{w}} d \bar{z}_{1}, e^{w} d z_{2}, e^{\bar{w}} d \bar{z}_{2}, d w, d \bar{w}
$$

As we consider $\mathfrak{g}_{\mathbb{C}}$ as a representation of $\mathfrak{g}$ via $\mathrm{Ad}$, we have the cochian complex $\Lambda \mathfrak{g}^{*} \otimes \mathfrak{g}_{\mathbb{C}}$ whose differential is given by

$$
\begin{aligned}
& d v_{1}=d w \otimes v_{1}, \quad d v_{2}=d \bar{w} \otimes v_{2}, \quad d v_{3}=-d w \otimes v_{3}, \quad d v_{4}=-d \bar{w} \otimes v_{4}, \\
& d v_{5}=-e^{-w} d z_{1} \otimes v_{1}+e^{w} d z_{2} \otimes v_{3}, \quad d v_{6}=-e^{\bar{w}} d \bar{z}_{1} \otimes v_{2}+e^{\bar{w}} d \bar{z}_{2} \otimes v_{4} .
\end{aligned}
$$

For $(w, 0,0) \in \mathbb{C}$, we have $\left(\operatorname{Ad}_{(w, 0,0)}\right)_{s}=\operatorname{diag}\left(e^{w}, e^{\bar{w}}, e^{-w}, e^{-\bar{w}}, 1,1\right)$ for the basis $\left\{v_{1}, \ldots, v_{6}\right\}$. Consider the cochain complex

$$
\left(\bigoplus_{\alpha} \bigwedge \mathfrak{g}^{*} \otimes V_{\alpha} \otimes V_{\rho}\right)^{\Phi(C)}
$$

as Section 5 where $C=\mathbb{C}$. Then we have

$$
\begin{aligned}
& \left(\bigoplus_{\alpha} \bigwedge_{\left.\mathfrak{g}^{*} \otimes V_{\alpha} \otimes V_{\rho}\right)^{\Phi(C)}}\right. \\
& \left.=\bigwedge^{-w} d z_{1} \otimes v_{e^{w}}, e^{-\bar{w}} d \bar{z}_{1} \otimes v_{e^{\bar{w}}}, e^{w} d z_{2} \otimes v_{e^{-w}}, e^{\bar{w}} d \bar{z}_{2} \otimes v_{e^{-\bar{w}}}, d w, d \bar{w}\right\rangle \\
& \quad \bigotimes\left\langle v_{1} \otimes v_{e^{-w}}, v_{2} \otimes v_{e^{-\bar{w}}}, v_{3} \otimes v_{e^{w}}, v_{4} \otimes v_{e^{\bar{w}}}, v_{5}, v_{6}\right\rangle
\end{aligned}
$$


For any lattice $\Gamma$ we have $b_{1}(G / \Gamma)=b_{1}(\mathfrak{g})=2$. But we will see that

$$
\operatorname{dim} H^{1}\left(G / \Gamma, E_{\text {Ad }}\right)
$$

varies for a choice of $\Gamma$. If $b, d \in \pi \mathbb{Z}$, then we have

$$
\begin{aligned}
A_{\Gamma}^{0}= & \left\langle v_{5}, v_{6}\right\rangle, \\
A_{\Gamma}^{1}= & \left\langle e^{-w} d z_{1} \otimes v_{1}, e^{-w} d z_{1} \otimes v_{e^{w}} \otimes v_{2} \otimes v_{e^{-\bar{w}}},\right. \\
& e^{-\bar{w}} d \bar{z}_{1} \otimes v_{e^{\bar{w}}} \otimes v_{1} \otimes v_{e^{w}}, e^{-\bar{w}} d \bar{z}_{1} \otimes v_{2}, \\
& e^{w} d z_{2} \otimes v_{3}, e^{w} d z_{2} \otimes v_{e^{-w}} \otimes v_{4} \otimes v_{e^{\bar{w}}}, \\
& e^{\bar{w}} d \bar{z}_{2} \otimes v_{e^{-\bar{w}}} \otimes v_{3} \otimes v_{e^{-w}}, e^{\bar{w}} d \bar{z}_{2} \otimes v_{4}, \\
& \left.d w \otimes v_{5}, d w \otimes v_{6}, d \bar{w} \otimes v_{5}, d \bar{w} \otimes v_{6}\right\rangle .
\end{aligned}
$$

Hence we have $\operatorname{dim} H^{1}\left(G / \Gamma, V_{\mathrm{Ad}}\right)=\operatorname{dim} H^{1}\left(A_{\Gamma}^{*}\right)=6$.

On the other hand, if $b \notin \pi \mathbb{Z}$ or $d \notin \pi \mathbb{Z}$, then we have

$$
\begin{aligned}
A_{\Gamma}^{0}= & \left\langle v_{5}, v_{6}\right\rangle, \\
A_{\Gamma}^{1}= & \left\langle e^{-w} d z_{1} \otimes v_{1}, e^{-\bar{w}} d \bar{z}_{1} \otimes v_{2}, e^{w} d z_{2} \otimes v_{3},\right. \\
& \left.e^{\bar{w}} d \bar{z}_{2} \otimes v_{4}, d w \otimes v_{5}, d w \otimes v_{6}, d \bar{w} \otimes v_{5}, d \bar{w} \otimes v_{6}\right\rangle .
\end{aligned}
$$

Hence we have $\operatorname{dim} H^{1}\left(G / \Gamma, E_{\mathrm{Ad}}\right)=\operatorname{dim} H^{1}\left(A_{\Gamma}^{*}\right)=2$.

\subsection{Dolbeault cohomology $H_{\bar{\partial}}^{*, *}(G / \Gamma)$}

For a coordinate $\left(z_{1}, z_{2}, z_{3}\right) \in \mathbb{C} \ltimes_{\phi} \mathbb{C}^{2}$, we consider the basis $\left(x_{1}, x_{2}, x_{3}\right)=$ $\left(d \bar{z}_{1}, e^{-\bar{z}_{1}} d \bar{z}_{2}, e^{\bar{z}_{1}} d \bar{z}_{3}\right)$ of $\mathfrak{g}^{*}$. We consider $C=\mathbb{C}=\left\{\left(z_{1}\right)\right\}$ and $\left(\alpha_{1}, \alpha_{2}, \alpha_{3}\right)=$ $\left(1, e^{z_{1}}, e^{-z_{1}}\right)$ for $C$ and $\left(\alpha_{1}, \alpha_{2}, \alpha_{3}\right)$ as in Section 5. If $b \notin \pi \mathbb{Z}$ or $c \notin \pi \mathbb{Z}$, then $(\star)$ holds and hence we have $H_{\bar{\partial}}^{*, *}(G / \Gamma) \cong \bigwedge \mathbb{C}^{3} \otimes H^{*}(\mathfrak{g})$. If $b, d \in \pi \mathbb{Z}$, then the condition $(\square)$ holds and hence we have $H_{\bar{\partial}}^{*, *}(G / \Gamma) \cong \bigwedge \mathbb{C}^{3} \otimes \wedge \mathbb{C}^{3}$. There exists a lattice $\Gamma$ which satisfies the condition $(\star)$ or $(\square)$ (see $[5]$ ).

\section{Acknowledgments}

The author would like to express his gratitude to Toshitake Kohno for helpful suggestions and stimulating discussions. This research is supported by JSPS Research Fellowships for Young Scientists. 


\section{References}

[1] L. Auslander, An exposition of the structure of solvmanifolds. I. Algebraic theory, Bull. Amer. Math. Soc. 79(2) (1973), 227-261.

[2] K. Dekimpe, Semi-simple splittings for solvable Lie groups and polynomial structures, Forum Math. 12(1) (2000), 77-96.

[3] K. Dekimpe, Solvable Lie algebras, Lie groups and polynomial structures, Composit. Math. 121(2) (2000), 183-204.

[4] N. Dungey, A.F.M. ter Elst and D.W. Robinson, Analysis on Lie Groups with polynomial growth, Birkhäuser (2003).

[5] K. Hasegawa, Small deformations and non-left-invariant complex structures on six-dimensional compact solvmanifolds, Differential Geom. Appl. 28(2) (2010), 220-227.

[6] A. Hattori, Spectral sequence in the de Rham cohomology of fibre bundles, J. Fac. Sci. Univ. Tokyo Sect. I, 8 (1960), 289-331 (1960).

[7] F. Hirzebruch, Topological methods in algebraic geometry, third enlarged ed., Springer-Verlag, 1966.

[8] J.E. Humphreys, Linear algebraic groups. Springer-Verlag, New York, 1981

[9] H. Kasuya, Minimal models, formality and hard Lefschetz properties of solvmanifolds with local systems, J. Differential Geom. 93(2) (2013), 269-298.

[10] H. Kasuya, Techniques of computations of Dolbeault cohomology of solvmanifolds, Math. Z. 273, (2013), 437-447.

[11] G.D. Mostow, Cohomology of topological groups and solvmanifolds, Ann. Math. (2) 73, (1961), 20-48.

[12] K. Nomizu, On the cohomology of compact homogeneous spaces of nilpotent Lie groups, Ann. Math. (2) 59, (1954), 531-538.

[13] A.L. Onishchik and E.B. Vinberg (Eds), Lie groups and Lie algebras II, Springer (2000).

[14] A. Polishchuk, Abelian varieties, theta functions and the Fourier transform. Cambridge University Press 2002. 
[15] M.S. Raghnathan, Discrete subgroups of Lie groups, Springer-Verlag, New York, 1972.

[16] Y. Sakane, On compact complex parallelisable solvmanifolds, Osaka J. Math. 13(1) (1976), 187-212.

[17] N. Steenrod, The topology of fibre bundles, Princeton University Press (1951).

Department of Mathematics

Tokyo Institute of TeChNology

1-12-1, O-OKAYAMA, MEguro

TOKYO 152-8551

JAPAN

E-mail address: kasuya@math.titech.ac.jp

Received December 2, 2013 
